Review

\title{
Aminoacyl-tRNA Synthetase Complexes in Evolution
}

\section{Svitlana Havrylenko ${ }^{1, \dagger}$ and Marc Mirande ${ }^{1,2, *}$}

1 Laboratoire d'Enzymologie et Biochimie Structurales (LEBS), CNRS, Université Paris-Sud, 1 avenue de la Terrasse, 91190 Gif-sur-Yvette, France

2 Institute for Integrative Biology of the Cell (I2BC), CEA, CNRS, Université Paris-Sud, 1 avenue de la Terrasse, 91190 Gif-sur-Yvette, France

$\dagger$ Present address: Institut Curie, CNRS UMR 168, Université Pierre et Marie Curie, 75248 Paris, France; E-Mail: svitlana.havrylenko@curie.fr.

* Author to whom correspondence should be addressed; E-Mail: marc.mirande@lebs.cnrs-gif.fr; Tel.: +33-1-69-823505; Fax: +33-1-69-823129.

Academic Editor: Michael Ibba

Received: 15 December 2014 / Accepted: 11 March 2015 / Published: 23 March 2015

\begin{abstract}
Aminoacyl-tRNA synthetases are essential enzymes for interpreting the genetic code. They are responsible for the proper pairing of codons on mRNA with amino acids. In addition to this canonical, translational function, they are also involved in the control of many cellular pathways essential for the maintenance of cellular homeostasis. Association of several of these enzymes within supramolecular assemblies is a key feature of organization of the translation apparatus in eukaryotes. It could be a means to control their oscillation between translational functions, when associated within a multi-aminoacyl-tRNA synthetase complex (MARS), and nontranslational functions, after dissociation from the MARS and association with other partners. In this review, we summarize the composition of the different MARS described from archaea to mammals, the mode of assembly of these complexes, and their roles in maintenance of cellular homeostasis.
\end{abstract}

Keywords: multi-aminoacyl-tRNA synthetase complexes; supramolecular assemblies; evolution; function 


\section{Introduction}

Decoding of genetic information is an essential step for all living organisms. The process of translation of the genetic message contained in mRNA into proteins is a universal mechanism conserved, with minor modifications, in the three branches of the tree of life, from bacteria, archaea, and to eukaryotes. A family of enzymes, the aminoacyl-tRNA synthetases, is responsible for pairing a specific amino acid to a cognate tRNA, thus establishing a univocal relationship between a triplet of nucleotides, the anticodon, and an elementary piece of proteins. Because the proper functioning of these enzymes is essential to gene expression, extensive biochemical and structural analysis of these proteins has been conducted, leading to a comprehensive view of this family of twenty enzymes [1,2]. In addition to their fundamental role in translation, aminoacyl-tRNA synthetases are also involved in other, unrelated noncanonical functions, such as regulation of gene expression, angiogenesis, and cellular signaling [3-5]. These secondary functions very often involve their association with cellular partners that are distinct from their regular partners in translation. When mutations affect the functioning of these enzymes, either in their translational or nontranslational functions, they have been associated with diseases in many cases [6-8]. Our understanding of the many implications of this family of enzymes thus requires a detailed knowledge of their mode of association with alternative partners.

Here, the analysis of aminoacyl-tRNA synthetase complexes is mainly restricted to the description of the complexes containing several synthetases, and does not address the transient or stable association of a single one of these enzymes with another protein (reviewed in [5]), such as the association of Escherichia coli ProRS with $\mathrm{YbaK}$, an editing domain appended in trans to several synthetases [9], of Methanocaldococcus jannaschii ProRS with HmdII, an inactive paralog of Hmd dehydrogenase that binds tRNA [10,11], or of Saccharomyces cerevisiae SerRS with Pex21p, a peroxisome biogenesis factor that strengthens the interaction of SerRS with its cognate tRNA [12]. During completion of this manuscript, an interesting review describing other complementary aspects of aminoacyl-tRNA synthetase complexes has been published [13].

\section{Multi-Aminoacyl-tRNA Synthetase Complexes (MARS)}

Early works provided some evidence for the existence of complexes containing several aminoacyl-tRNA synthetases in the bacteria E. coli [14], in the yeast $S$. cerevisiae [15], or in rat liver [16]. These authors described the occurrence of high-molecular-mass aminoacyl-tRNA synthetases in crude extracts of cells after analysis by chromatography on agarose columns or by centrifugation in sucrose gradients, but also noticed that these assemblies were fragile and highly prone to dissociation. In the light of recent data showing that aminoacyl-tRNA synthetases, and more generally components of the translation apparatus, are able to transiently interact with cellular components such as polysomes or actin filaments [17-19], the finding that some components are coeluting as large entities is not sufficient to ascertain that they form complexes. The occurrence of aminoacyl-tRNA synthetases within complexes of defined composition, which are not the result of subcellular interactions with filamentous structures such as mRNA or actin and tubulin polymers and 
involve direct protein-protein interactions between partners, requires isolation and characterization of the complexes.

Here, the composition of the MARS isolated and characterized in different species is presented, with the aim to identify the rules governing the assembly of these enzymes and the possible involvement of additional factors in this process.

\subsection{Complexes in Archaea}

It was long believed that association between aminoacyl-tRNA synthetases to form complexes is restricted to eukaryotic cells. However, analysis of the structural organization of synthetases in archaea provided compelling evidence for the occurrence of multi-aminoacyl-tRNA synthetase complexes.

In the archaea Methanothermobacter thermoautotrophicum, a complex containing LeuRS, LysRS and ProRS has been isolated (Figure 1) [20]. The dissociation constants determined for association of LeuRS with LysRS and LeuRS with ProRS were in the micromolar range, suggesting the assembly of a stable complex. Structural mapping of this complex indicated that LeuRS plays the role of a scaffolding protein, the core, $N$-terminal domain of LeuRS binding LysRS and its $C$-terminal domain binding ProRS [21]. In addition, elongation factor EF1A, which forms a ternary complex with GTP and aminoacyl-tRNA to deliver aatRNA to the ribosome, also associates with the CP1 proofreading domain of LeuRS [22]. Stable association $\left(K_{\mathrm{D}}\right.$ of $\left.250 \mathrm{nM}\right)$ of SerRS and ArgRS from the same organism (Figure 1) was also reported to improve their catalytic activity at high temperature [23]. These two synthetases interact with the ribosome in the region of the L7/L12 stalk [24].

In another species of archaea, Thermococcus kodakarensis, affinity purification of LeuRS resulted in the isolation of several components of the translation machinery that were identified by mass spectrometry, suggesting that LeuRS forms a complex with TyrRS and ProRS [25]. This complex may also interact with EF1A and ribosomes. However, direct interaction between all these components through protein-protein interaction remains to be established.

A characteristic feature of all the MARS described so far in archaea, is that they do not possess auxiliary proteins involved in their assembly. The scaffold protein of the MARS in M. thermoautotrophicum is LeuRS, one the synthetase components [21]. This is a distinctive feature of these complexes, as compared to those found in eukaryotes.

\subsection{Complexes in Unicellular Eukaryotes}

\subsubsection{The MARS in Saccharomyces cerevisiae}

In the yeast Saccharomyces cerevisiae, MetRS forms a complex with GluRS via association with Arc1p, a protein homologous to the p43 component of the MARS found in mammals (Figure 1) [26]. Inactivation of Arc1p by gene disruption in yeast is not lethal but results in slow growth. In the MetRS-Arc1p-GluRS complex, Arc1p has two functions. First, it plays a role of cofactor for the two synthetases. The $C$-terminal domain of Arclp is a tRNA-binding protein. Association of Arclp with MetRS and GluRS stimulates the tRNA-aminoacylation activity of the two synthetases. The levels of stimulation of aminoacylation activity range from 400-fold [27] to 4-fold [28] for MetRS, and is about 2-fold for GluRS [29] in the presence of Arc1p. In yeast, S. cerevisiae Arc1p can be replaced by 
human $\mathrm{p} 43$, a protein that does not interact with $S$. cerevisiae MetRS and GluRS, suggesting that Arc1p is also involved in the sequestration of tRNA in the cytoplasm to increase its local concentration [30]. Second, the intracellular location of the two synthetases rests on the assembly/disassembly of the complex with Arclp. In the absence of Arc1p, MetRS is redistributed to the nucleus [31,32] and GluRS to the mitochondria where it synthesizes Gln-tRNA ${ }^{\text {Gln }}$ via the GatFAB-dependent transamidation pathway [33]. The role of nuclear MetRS remained unclear until the recent discovery that it regulates the expression of genes involved in oxidative phosphorylation in mitochondria, in an unexpected concerted manner between nuclear and mitochondrial genes [32].

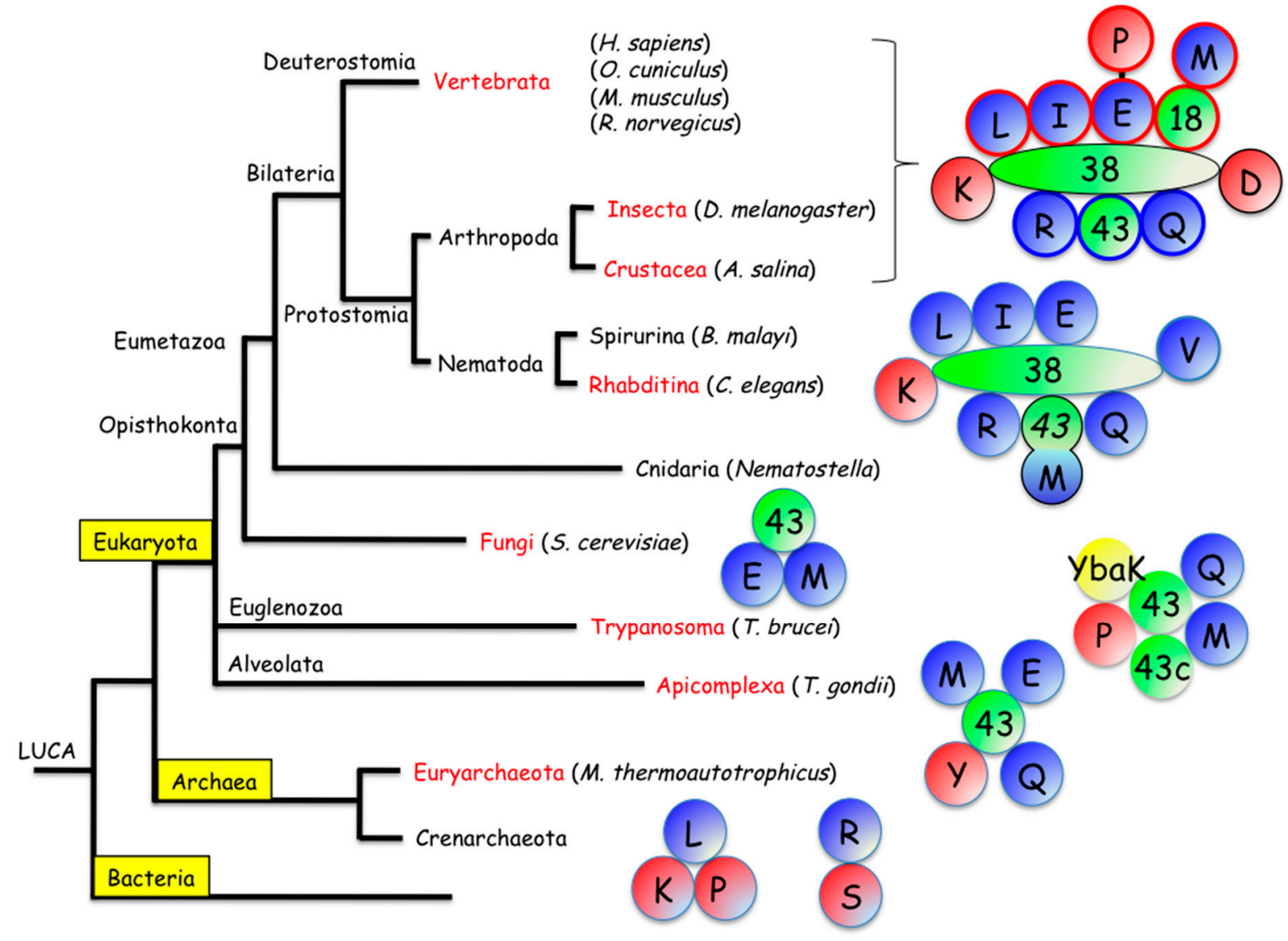

Figure 1. Occurrence of multi-aminoacyl-tRNA synthetase complexes of different compositions throughout the tree of life. A schematic view of the complexes described in Euryarchaeota, Apicomplexa, Trypanosoma, Fungi, Rhabditina, Crustacea, Insecta, or Vertebrata, is shown. The auxiliary proteins known to have a structural role within the complexes are presented in green. Class I synthetases are in blue; class II in red. Synthetases are indicated according to the one letter symbol of their amino acid substrate. The autonomous editing protein $\mathrm{YbaK}$ is in yellow. On the right are represented the complexes described in the literature. The complex isolated from vertebrata is composed of a scaffolding protein (p38), that joins sub-complex I (components circled in red), subcomplex II (components circled in blue), LysRS (K) and AspRS (D). In the complex from Rhabditina, the p43 and MetRS (M) proteins are fused in a single polypeptide. The scaffold protein of the complexes from Apicomplexa and Trypanosma have characteristics of $\mathrm{p} 43$ from vertebrates. 
Saccharomyces cerevisiae is able to grow on either fermentable (glucose) or nonfermentable (ethanol) carbon sources. When cells are switched from fermentation to respiratory metabolism, the oxidative phosphorylation pathway in mitochondria is stimulated to increase ATP production. In particular, the $\mathrm{F}_{1} \mathrm{FoATP}_{\mathrm{T}}$ synthase complex is overexpressed. This complex is made of several subunits that are either expressed in mitochondria, or are encoded by the nuclear genome. It has been shown that dissociation of the MetRS-Arc1p-GluRS complex is responsible for the synchronized expression of the various subunits of the $\mathrm{F}_{1} \mathrm{~F}_{\mathrm{O}} \mathrm{ATP}$ synthase complex [32]. During the transition from fermentation to respiration, expression of Arc1p is downregulated via the Snfl/4 pathway, leading to the release of both MetRS and GluRS from the MetRS-Arc1p-GluRS complex [32]. GluRS is at least in part relocated to the mitochondria in response to a higher demand in the translation of the mitochondria-encoded subunits of the $\mathrm{F}_{1} \mathrm{FOATP}_{\mathrm{O}}$ synthase complex [33], and MetRS is translocated to the nucleus where it participates in transcription of nuclear ATP synthase genes via a still uncharacterized mechanism [32]. Because MetRS and GluRS are essential enzymes for cytosolic translation, it would be interesting to get more insight on the extent of GluRS and MetRS relocation during the diauxic shift, and on the reverse mechanism after transition from respiration to fermentation growth conditions.

Genetic studies suggested that the $N$-terminal domain is necessary and sufficient for binding the $N$-terminal appended domains of MetRS and GluRS [31]. The crystal structure of two binary complexes made of the N-domain of Arc1p in complex with either the N-domain of MetRS or the $\mathrm{N}$-domain of GluRS, suggested a possible mode of assembly of this complex [34]. The $N$-terminal domains of the three proteins adopt a GST (glutathion S-transferase)-like fold. Whereas classical GST proteins are dimers, Arclp is a monomer in solution [28]. While the geometry of interaction between Arc1p and MetRS is reminiscent of a canonical GST dimer, Arclp and GluRS also interact via their GST-like moieties but use different protein interfaces, allowing Arc1p to simultaneously bind MetRS and GluRS [34]. The assembly of the complex solely rests on these interactions, since site-directed mutagenesis of residues located at the interfaces, or deletion of these domains result in cellular relocation of the two synthetases [31,32,35]. Arc1p is a target of Blp1 in yeast, a biotin:protein ligase, but biotinylation of Arc1p at $\mathrm{Lys}^{86}$, within its $N$-terminal domain, has no effect on the assembly of the MetRS-Arc1p-GluRS complex [36]. The structure of the ternary complex is highly dynamic in solution, as revealed by small-angle X-ray scattering analyses conducted in the absence or in the presence of $\mathrm{tRNA}^{\mathrm{Met}}$ and $\mathrm{tRNA}^{\mathrm{Glu}}$ [37]. The radius of gyration of the ternary MetRS-Arclp-GluRS complex is of $97 \AA$, as compared to $60 \AA$ for the pentameric complex obtained after addition of tRNA. This suggests that the activity of Arclp as a cofactor for binding tRNA to the active site of the two synthetases requires large conformational changes resulting in a compaction of the complex. These data also point to a large flexibility of the complex, a characteristic that hinders its structural study at high resolution.

\subsubsection{The MARS in Toxoplasma gondii}

Toxoplasma gondii is an intracellular parasite, a member of the phylum of Apicomplexa, corresponding to unicellular eukaryotes. In Toxoplasma, a protein named Tg-p43 shares about 35\% sequence similarity with human $\mathrm{p} 43$, especially in its $C$-terminal domain, which is identified as the 
tRNA-binding EMAPII domain. This observation suggested that a MARS is present in Toxoplasma. Using this p43-like protein as a bait, a complex containing MetRS and GluRS (as found in S. cerevisiae), but also GlnRS and TyrRS was isolated (Figure 1) [38]. Similarly to the MetRS-Arclp-GluRS complex in yeast, the N-domain of Tg-p43 was sufficient to form the complex. Deletion of Tg-p43 is not lethal for Toxoplasma, and does not alter its pathogenicity. Initial electron microscopy imaging of the complex suggests a large degree of flexibility of the particle around a central ring-like core.

\subsubsection{The MARS in Trypanosoma brucei}

Trypanosoma brucei is another parasite belonging to the large phylum of flagellate protozoa. TAP-tagged MetRS, ProRS, IleRS and MCP2, a protein sharing similarities with human p43, were copurified with several components of the translation machinery that were identified by mass spectrometry [39]. Globally, three aminoacyl-tRNA synthetases (MetRS, ProRS, GlnRS) could form a core complex with MCP1 and MCP2 (MARS complex-associated proteins 1 and 2), two proteins related to human $\mathrm{p} 43$, and $\mathrm{MCP}$, a protein showing similarities with $\mathrm{YbaK}$, a free-standing editing domain (Figure 1). MCP-2 only contains the $C$-terminal binding domain found in yeast Arc1p and in human $\mathrm{p} 43$, and MCP1 also contains the GST-like $N$-terminal domain of full-length Arc1p and p43. AlaRS, TrpRS and AspRS, but also CysRS, GluRS, GlyRS and IleRS could be more loosely associated to the core complex. MCP2 enhances tRNA aminoacylation catalyzed by the associated enzymes, and repression of MCP2 in T. brucei reduces parasite growth and infectivity in mice. More detailed biochemical and structural characterization of this MARS would help to clarify its composition, structural organization and function.

\subsection{The MARS in Eumetazoa}

\subsubsection{The MARS in Deuterostomia}

\section{Composition of the MARS}

The aminoacyl-tRNA synthetase complex that was first isolated is the MARS from vertebrates. Co-isolation of several aminoacyl-tRNA synthetases is invariably observed when synthetases are purified from rat liver, rabbit liver or reticulocytes, sheep liver and human placenta [40-47], or from mammalian cells in culture [48,49]. It contains the nine synthetases specific for the amino acids Arg, Asp, Gln, Glu, Ile, Leu, Lys, Met and Pro (Figure 1). In addition, three additional proteins always copurify with the particulate synthetases, p18 [50], p38 [51] and p43 [52]. One of the synthetase components contains two aminoacyl-tRNA synthetases fused on a large, single polypeptide chain of $171 \mathrm{kDa}$, corresponding to glutamyl-prolyl-tRNA synthetase (GluProRS) [53,54]. The two-synthetase domains are separated by a linker region made of three repeated sequence motifs of about 50 amino acids, named the WHEP domains because they were discovered in human TrpRS (W), HisRS (H), and GluProRS (EP). Their occurrence in human MetRS and GlyRS was also reported later on. This polypeptide arose from a gene fusion event [55,56] occurring more than 1 billion years ago in Eumetazoa, after divergence from Fungi [57]. This complex contains monomeric (ArgRS, GlnRS, IleRS, LeuRS, MetRS) or dimeric enzymes (AspRS, GluProRS, LysRS) for a mass of about 1.5 MDa 
determined by SEC-MALS analysis [58]. Among these components, the same gene encodes two distinct forms of ArgRS, GluRS and LysRS. In addition to the $74 \mathrm{kDa}$ species of ArgRS present in the MARS, another form of $60 \mathrm{kDa}$ is produced by alternative translation initiation on a second ATG on the same mRNA [59,60]. ArgRS from the MARS delivers Arg-tRNA for translation, and Arg-tRNA produced from the free form is believed to deliver tRNA for arginylation of proteins in the ubiquitin-dependent degradation pathway [61,62]. Two copies of GluProRS are present in the MARS [58], and a truncated form of GluProRS containing only the GluRS moiety was identified [63]. This GluRS-only species is expressed from the same gene after polyadenylation-directed conversion of a Tyr codon to a stop codon. It is not known whether this GluRS participates in protein translation in the cytoplasm. Concerning LysRS, a single gene encodes the cytoplasmic and mitochondrial enzymes which are produced by alternative splicing of exon 2 of the KARS gene [64]. The mRNA with a deletion of sequences encoded by exon 2 expresses the cytoplasmic species of LysRS produced by translation initiation in mRNA sequences encoded by exon 1. The cytoplasmic species of LysRS is the only one to be present in the MARS complex [65]. The reason for the selection of these nine synthetases and only those nine is unclear but a correlation with the size of their amino acid substrates [66] or with their connection with intermediates of the citric acid cycle [67] has been proposed.

The three auxiliary proteins have an essential structural role for the assembly of the MARS, and also fulfill important functions in the translation machinery. The p38 protein of the MARS is a dimeric protein made of 320 amino acid residues with no homolog in yeast, bacteria and archaea. It has the potential to interact with several components of the MARS and was thus identified as the scaffold protein of the complex [51]. Its $C$-terminal moiety is a GST-like domain, and its $N$-terminal region contains a leucine zipper motif $[51,68]$. A splice variant of $\mathrm{p} 38$ with a deletion of exon 2 , expressing a protein with a deletion of the leucine zipper region, does not associate within the MARS and promotes tumorigenesis via degradation of p53 [69]. Assembly of the MARS is severely impaired after shRNA-mediated knockdown of p38 in HeLa cells [70], or after introduction of mutation within the structural gene of p38 in mice [71]. In neurons, a balanced expression of p38 with other components of MARS is regulated by ubiquitin-dependent degradation of p38, which involves Parkin, an E3 ubiquitin-protein ligase. Abnormal accumulation of p38 in dopaminergic neurons is linked to Parkinson's disease, due to progressive neurodegeneration [72-74]. The p18 component is a small protein of 174 amino acid residues [50]. The crystal structure reveals a GST-like fold similar to that observed for the yeast Arc1p component of the MetRS-Arc1p-GluRS complex [75]. It promotes association of MetRS within the MARS [70] and is also involved in transfer of Met-tRNA ${ }_{i}{ }^{\text {Met }}$ to eukaryotic initiation factor 2 (eIF2) [76]. The p43 component has homology with yeast Arc1p [52]. The native protein has a strong tRNA-binding capacity $\left(K_{\mathrm{D}}\right.$ of $\left.0.2 \mu \mathrm{M}\right)$ that is lost upon cleavage with caspase 7 which generates endothelial-monocyte-activating polypeptide II (EMAPII) [77], an inflammatory cytokine released under apoptotic conditions [78]. The EMAPII domain is similar to bacterial tRNA-binding protein Trbp111 [79] and forms an oligonucleotide-binding (OB) fold [80,81]. Its $N$-terminal moiety anchors the procytokine to the MARS and is required for association of GlnRS and ArgRS to the complex [70]. The tRNA-binding domain of p43 is supposed to act as a cofactor in trans for the binding of tRNA by one or several synthetases of the MARS. A role of p43 in the delivery of tRNA to the ArgRS component of MARS was proposed [82], but another study did not confirm these data [83]. Interestingly, two p43 proteins of different lengths are encoded by the same 
gene and produced by the same mRNA [84]. Translation initiation from two in-frame AUGs generates mitochondrial and cytoplasmic forms of $\mathrm{p} 43$. The longest translation product contains nine additional $N$-terminal amino acid residues, which correspond to a mitochondrial targeting sequence (MTS). The function of $\mathrm{p} 43$ in mitochondria is not known.

Assembly of the MARS

In vertebrates, highly purified preparations of the MARS contain eleven polypeptides with molecular masses ranging from $171 \mathrm{kDa}$ for GluProRS to $20 \mathrm{kDa}$ for p18 (Figure 2) [58]. Structural mapping of the complex to determine topological relationships between the various components has been conducted by different approaches: reverse chemical crosslinking [85], extensive two-hybrid searches for pairs of interacting partners [51,86], pull-down experiments of native proteins, of truncated derivatives or of fragments to identify the regions of the two partners involved in protein-protein interactions [83,87-89], assembly of subcomplexes with purified individual components [89], or knockdown of the non-essential p18, p38 and p43 proteins in cultured cells that induced perturbations of the assembly of the complex [70]. Although many of the reported data were contradictory, a general scheme of complex assembly could be proposed, which reconciles most of the published data (Figure 2). The MARS is made of two subcomplexes linked by the p38 scaffold protein [51,70,71]. Sub-complex 1 contains GluProRS, IleRS, LeuRS to which MetRS is also associated via the auxiliary protein $\mathrm{p} 18$. Sub-complex 2 associates ArgRS and GlnRS with p43. LysRS and AspRS are direct interactors of p38. The complete network of interactions results in a stable particle that cannot be easily dissociated under nondenaturing conditions. Binding affinities in the range of $0.3 \mathrm{nM}$ to more than $100 \mathrm{nM}$ were determined for association of LysRS with 38, or p43 with ArgRS, respectively [89].

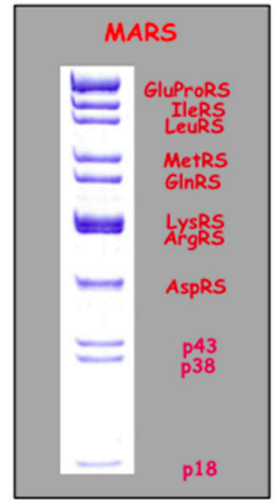

Purified MARS

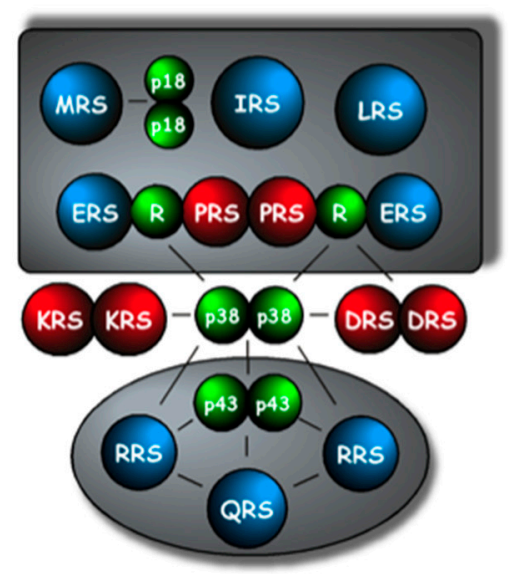

Protein:protein interaction map

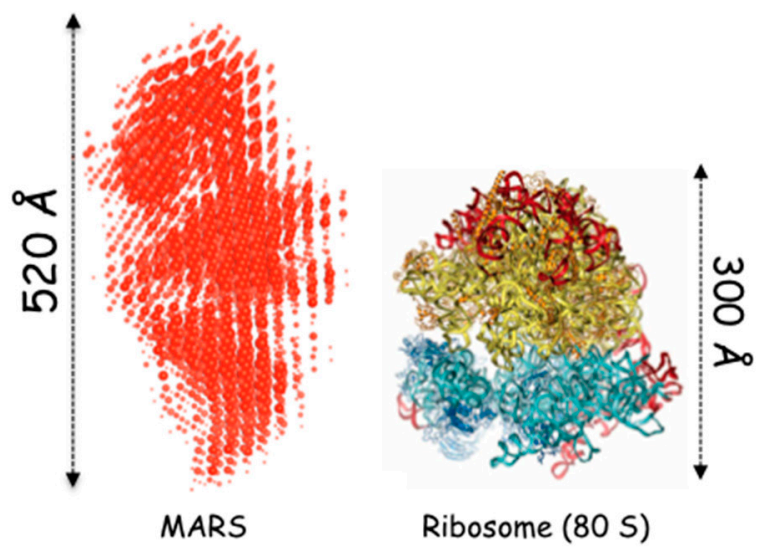

$3 D$ architecture of MARS

Figure 2. Composition, structural organization and 3D-architecture of the MARS. Left: SDS-PAGE analysis of the MARS from rabbit; Middle: protein:protein interaction map of the MARS, showing two subcomplexes linked by a scaffold protein, p38; Right: low resolution envelope of the MARS determined by SAXS (Small Angle X-ray Scattering). The crystal structure of the $80 \mathrm{~S}$ ribosome is shown for comparison. 
Association of synthetases to the MARS either involves the appended eukaryote-specific domains or the core domain of the components. The GST-like $N$-terminal appended domain of MetRS that interacts with p18 [90-92], the leucine-rich $N$-terminal appended domain of ArgRS that interacts with p43 [83], the $C$-terminal appended domains of LeuRS [87] and IleRS [88], and the GST-like $N$-terminal appended domain of GluProRS, are required for complex assembly. Concerning LysRS, AspRS and GlnRS, the core domains of the synthetases are believed to be involved in complex assembly [93,94]. Only few structural data are available at high resolution to describe the protein:protein interaction sites. LysRS associates with the very $N$-terminal region of p38 [51]. The crystal structure of a complex made of a $N$-terminally truncated form of LysRS and of a $N$-terminal peptide of p38 reveals the mode of association of these two proteins [95]. The site of interaction with p38, which identifies the site of interaction within the MARS, is located underneath the LysRS dimer, leaving the other side fully accessible for binding and aminoacylating tRNA. The solution structure of the leucine zipper of p38, which interacts with the leucine zipper of $\mathrm{p} 43$, has also been reported but does not provide precise structural information about organization of the p38:p43 complex [68].

The assembly of subcomplex II of MARS has been extensively studied. The $N$-terminal appended domain of ArgRS is essential for its assembly within the MARS [60,61]. It mediates its association with p43 [83]. ArgRS and p43 are required for association of GlnRS to subcomplex II [89]. The crystal structure of subcomplex II reveals that the $N$-terminal domain of ArgRS is a long $\alpha$-helix, which forms a coiled-coil with the $N$-terminal helix of $\mathrm{p} 43$, and interacts with the catalytic core of GlnRS [96]. This first high-resolution structure of a subcomplex of MARS, showing an elongated arrangement (100 $\AA$ in length), is a significant breakthrough in the field, but many questions are left open. Only one molecule of each ArgRS, GlnRS and p43 are present in the asymmetric unit of the crystal. The hexameric complex deduced from a twofold symmetry operation could represent the native structure of subcomplex II, but this conformation does not allow access of tRNA to the catalytic core of ArgRS. This suggests that activity of ArgRS within the complex requires a large conformational change. Interaction between the long $N$-terminal helical domain of ArgRS and $\mathrm{p} 43$ is also supposed to be essential for the activity of ArgRS [96], but earlier results showed that full-length ArgRS and a truncated derivative lacking this $N$-terminal domain have similar kinetic parameters in the tRNA aminoacylation reaction [97].

Low-resolution models of the MARS, obtained by electron microscopy and three-dimensional reconstructions [98] or by small-angle X-ray scattering (SAXS) in solution (Figure 2) [58], reveal a large particle with external dimensions ranging from $25 \mathrm{~nm} \times 30 \mathrm{~nm} \times 23 \mathrm{~nm}$ [98] to an elongated molecule of about $50 \mathrm{~nm}$ in length [58]. As discussed in this paper, the model of MARS deduced from electron microcopy analyses may represent only a subset of the particle. The multi-armed shape of MARS observed by SAXS certainly reflects the flexibility of the different domains of the complex. This non-compact structural organization of MARS could be appropriate to allow large structural rearrangements upon tRNA binding, as observed for the yeast MetRS-Arclp-GluRS complex [37]. This open structure may also favor efficient release of the components that have been reported to fulfill non-canonical functions after dissociation from the complex. It would be now interesting to see how the crystal structure of subcomplex II could be fitted into the low-resolution envelope of MARS described by SAXS. 


\subsubsection{The MARS in Protostomia}

Arthropoda

The MARS was described in two members of the Arthropoda family (Figure 1), in the Insecta Drosophila melanogaster [99] and in the Crustacea Artemia salina [100]. The complex purified from the fly $D$. melanogaster contains the same nine aminoacyl-tRNA synthetases and the same three auxiliary proteins, as found in mammals [99]. Its structural organization was not analyzed, but is believed to be very close to that reported for the MARS in Deuterostomia. The main differences concern GluProRS, which contains six WHEP domains instead of three in the inter-synthetase region, and MetRS, which contains three WHEP domains appended at the $C$-terminus instead of one. The p43, p38 and p18 proteins share 43\%, 21\% and 30\% identity with the corresponding proteins from human MARS. Because the same MARS was found in Deuterostomia, represented by the MARS isolated from mammals, and in Protostomia, represented by the MARS from the fly D. melanogaster, the two branches of Bilateria, it was tempting to speculate that this type of MARS was already present at the origin of Bilateria.

\section{Nematoda}

Nematoda is one of the other main branches of Protostomia (Figure 1). The MARS from Caenorhabditis elegans, a member of the Rhabditina sub-branch of Nematoda has been isolated. Aminoacyl-tRNA synthetases from the worm C. elegans display a high level of similarity with the human counterparts. Sequence comparison of the synthetases from the MARS of human with homologous proteins from $C$. elegans suggested that some of these enzymes had a high probability to be found in a MARS, whereas some other did not show characteristics of the proteins associated in the human MARS (Figure 3). The core region of the synthetases, corresponding to the catalytic and anticodon-binding domains, which share from 47\% identities for ArgRSs to 59\% identities for AspRSs, are generally well conserved between C. elegans and human, but eukaryote-specific appended domains are remarkably distinct for some pairs of synthetases [101]. AspRS, GlnRS, LeuRS and LysRS from $C$. elegans, which display an extensive global conservation with their human homologs were likely to be found in the MARS of $C$. elegans, together with ArgRS which displays the leucine-rich $\mathrm{N}$-terminal extension involved in the assembly of human ArgRS in the MARS. On the contrary, the possible association of IleRS, MetRS, GluRS and ProRS in a complex in C. elegans was questionable. IleRS from $C$. elegans does not share the two $C$-terminal repeated domains of human IleRS; MetRS in $C$. elegans has a very distinct structural organization, without a $N$-terminal GST-like domain and with a p43-like $C$-terminal domain [102]; and GluRS and ProRS do not form a fused protein in C. elegans (Figure 3). In addition, no protein homologous to p18 or to p43 could be identified in $C$. elegans. Only a very putative p38-like protein with $16 \%$ identities with human p38 was reported.

Surprisingly, the MARS isolated from $C$. elegans contains seven of the nine synthetases found in the human MARS, namely ArgRS, GlnRS, GluRS, IleRS, LeuRS, LysRS and MetRS, but does not contain AspRS and ProRS [101]. The p38-like protein is essential for the assembly of the synthetases. In a mutant strain of $C$. elegans with a deletion of the $67 C$-terminal residues of p38, several 
synthetases are released from the complex [101]. Association of MetRS to this complex is not mediated by association in trans with an auxiliary protein, as in the case of p18 in the human MARS, but by fusion in cis of a p43-like domain, suggesting that bona fide p43 and MetRS genes fused in Rhabditina. At the same time, a fission event occurred in the GluProRS gene [55], leaving only its GluRS moiety attached to the complex via its $N$-terminal GST-like domain.

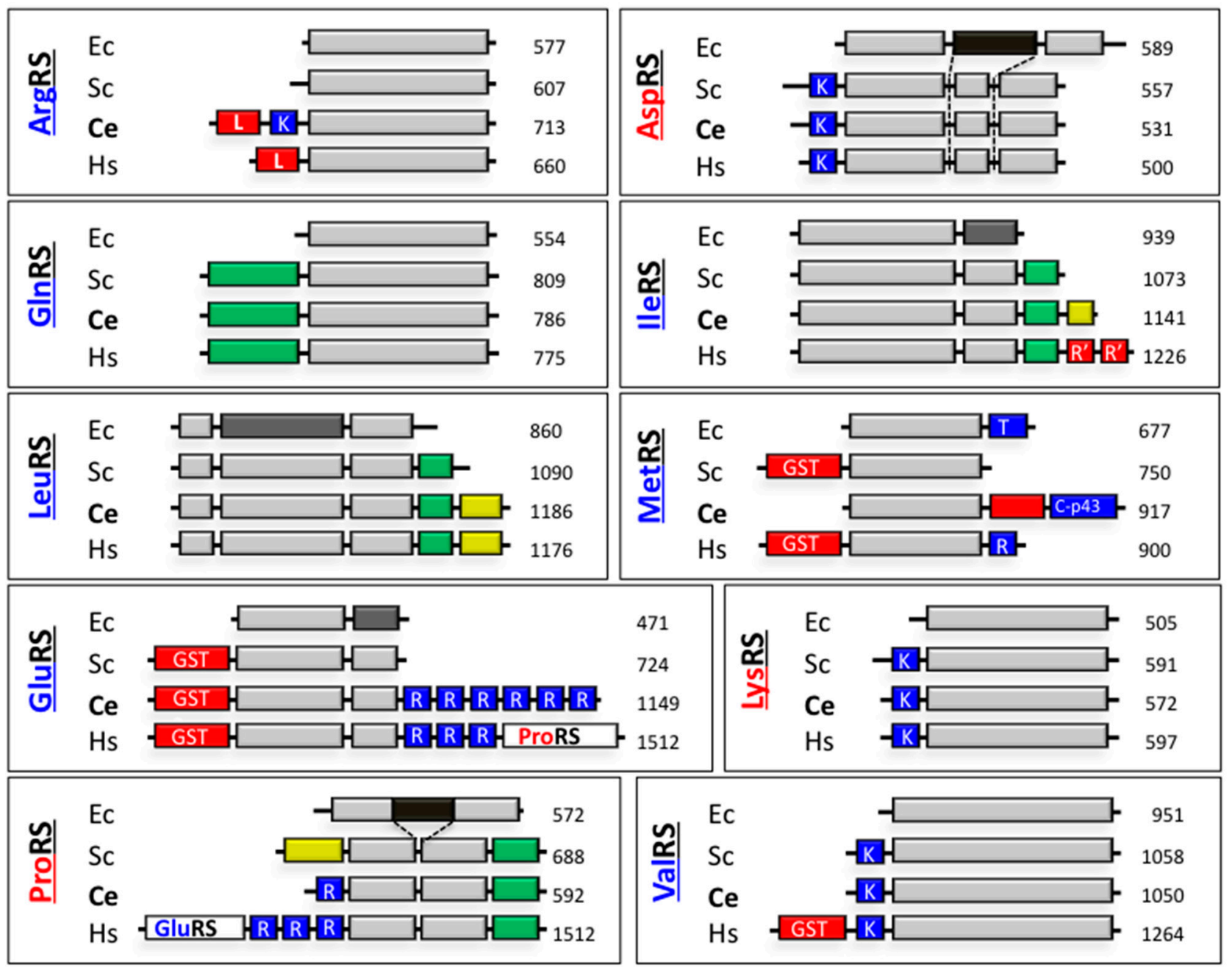

Figure 3. Schematic comparison of ten aminoacyl-tRNA synthetases from $H$. sapiens (Hs) with their homologs in E. coli (Ec), S. cerevisiae (Sc) and C. elegans (Ce). Conserved parts are shown in light grey with poorly conserved regions in medium grey. Dark grey boxes represent E. coli specific domains. The number of amino acid residues per polypeptide chain is indicated. Eukaryote specific domains, conserved from yeast to human, are shown in green. Appended domains found only in one or two species are in yellow. Protein-protein and protein-RNA interaction domains are shown in red and blue, respectively: L-leucine-rich domain; GST-glutathion $S$-transferase-like domain; $\mathrm{R}$ '- repeated sequence found in human IleRS; K-lysine-rich domain; R-repeat (WHEP) domain. The names of class I aaRS are highlighted in blue, of class II in red.

Unexpectedly, ValRS is associated in the MARS complex of C. elegans. This enzyme shares the $N$-terminal tRNA-binding domain found in yeast and human ValRS, but does not possess the GST-like 
$N$-terminal domain of the human enzyme (Figure 3). In Vertebrata, ValRS forms a complex with elongation factor EF1A, and with the three subunits of its guanine nucleotide exchange factor, $\mathrm{EF} 1 \mathrm{~B} \alpha$, EF1B $\beta$ and EF1B $\gamma$ [103-105]. The $N$-terminal GST-like domain of ValRS is indispensable for its interaction with the EF1B $\beta$ subunit of the EF1 complex [106]. Analysis of ValRS sequences in Eukaryota reveals that the presence of this GST-like protein-binding domain (PBD), and thus the propensity of ValRS to associate with the EF1A/EF1B complex, could be restricted to the branch of Deuterostomia from Bilateria (Figure 4). In the absence of this domain, association of ValRS with the MARS does not appear to be a general feature of ValRS since no stable association of ValRS was reported with other MARS, especially in the sister group of Arthropoda. Therefore, sequence-based analysis of the synthetases is certainly not sufficient to predict with high confidence their ability to form supramolecular complexes with other synthetases, as in the case of the MARS, or with other components of the translation apparatus such as EF1A/EF1B.

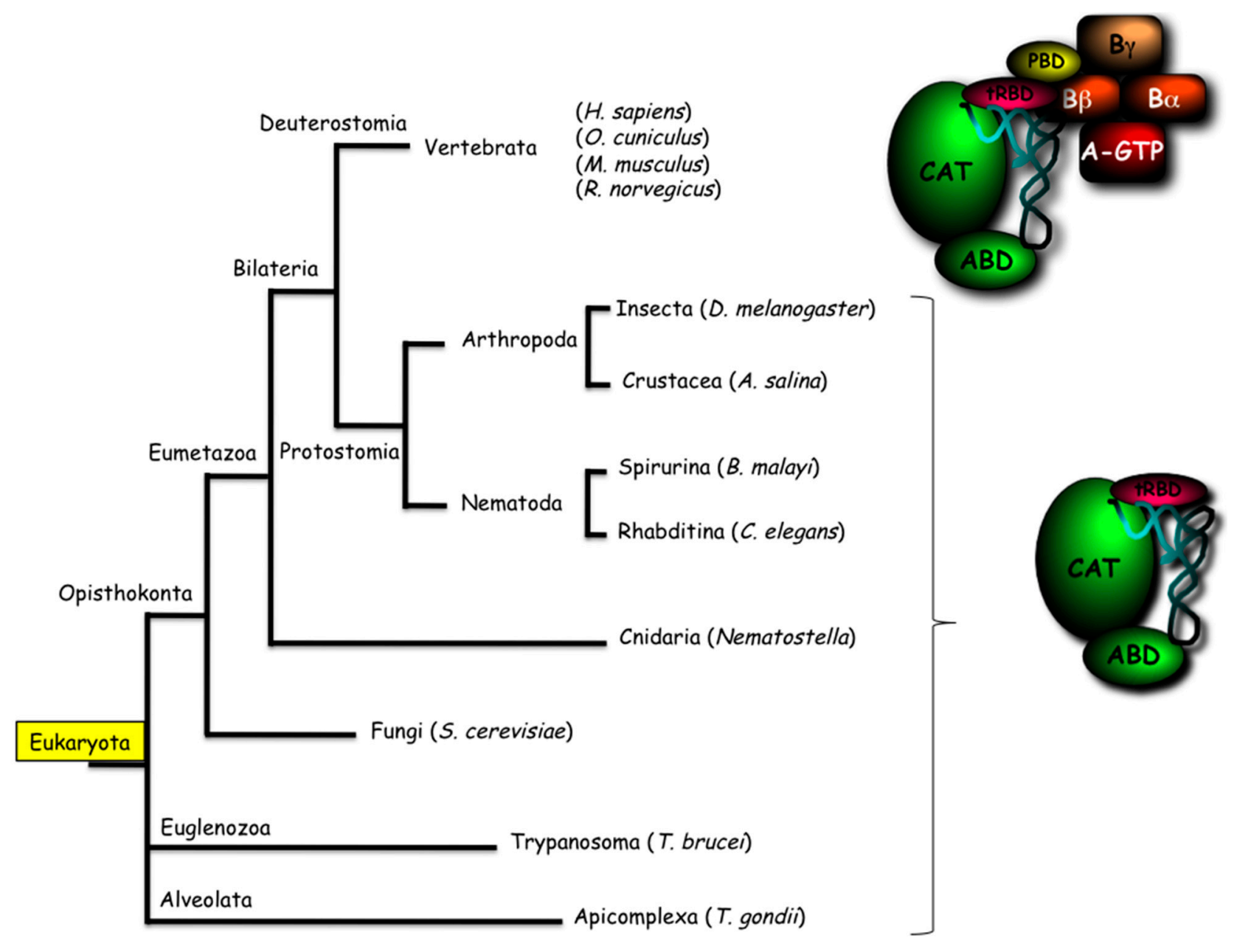

Figure 4. Occurrence of ValRS:EF1A:EF1B complexes or of "free" ValRS in Eukaryota. The conserved catalytic (CAT) and anticodon-binding (ABD) domains are in green. The appended tRNA-binding domain (tRBD) is indicated in red, the protein-binding domain (PBD) only recovered in Deuterostomia, required for association with elongation factor 1 , is in yellow. 


\section{Integration of the MARS in Cellular Homeostasis}

\subsection{Integration of the MARS in Translation Apparatus}

Association of the MARS with elongation factor EF1A and with ribosomes/polysomes has often been reported. The multisynthetase complexes described in archaea have been suggested to interact with EF1A and with ribosomes to facilitate cycling of tRNAs in translation [22,24,25]. In eukaryotes, interaction of the MARS with EF1A, ribosomes and polysomes has also been reported [17,107,108]. Association of components of the translation machinery with the actin filamentous network has been reported [17,109]. The working model assumes that tRNA is never free in the cytoplasm of eukaryotic cells, but is vectorially transferred from the synthetase, to elongation factor EF1A, to ribosome, and back to the synthetase $[19,110]$ suggesting that the process of tRNA cycling in translation is processive [111]. This implies that stable and transient protein:protein, and protein:nucleic acid interactions are essential to ensure proteostasis.

\subsection{Role of the MARS in Regulation of Other Cellular Functions}

In addition to their essential role in translation, several components of the MARS are also involved in nontranslational functions, which were recently described in several excellent reviews [6-8]. Only a few examples are summarized below. These noncanonical functions are related to cell signaling and control of cellular homeostasis, and especially to the regulation of fundamental physiological processes such as inflammation, angiogenesis and tumorigenesis. Association/dissociation of components of the MARS could be a means to regulate in space and in time their activity in translational or nontranslational processes [112].

In some cases it has been clearly shown that dissociation from the complex is related to a gain of new functions. After phosphorylation on $\mathrm{Ser}^{207}$ in stimulated mast cells, LysRS dissociates from the MARS, translocates to the nucleus and stimulates transcription of genes involved in the immune response via activation of microphtalmia-associated transcription factor MITF [95,113,114]. This transcriptional function of a synthetase rests on the ability of LysRS to synthesize diadenosine tetraphosphate $\left(\mathrm{Ap}_{4} \mathrm{~A}\right)$ when, in the absence of tRNA, aminoacyl-adenylate can react with a second molecule of ATP [115]. Several aminoacyl-tRNA synthetases from bacteria to mammals, in addition to LysRS, have the ability to synthesize Ap4A, which suggests that they may be involved in the regulation of various cellular processes. After phosphorylation of the WHEP domains in IFN $\gamma$-stimulated cells, GluProRS dissociates from the MARS, and associates with components of the GAIT ( $\gamma$-interferon activated inhibitor of translation) complex to induce translational silencing of inflammation-related mRNAs [116,117]. Laminin is a major constituent of the extracellular matrix that interacts with receptors and induces cell migration. After laminin stimulation, LysRS is phosphorylated at $\mathrm{Thr}^{52}$ by p38 mitogen-activated protein kinase (MAPK), translocates to the plasma membrane, interacts with the 67LR receptor, and promotes metastasis [118]. Cleavage of the p43 component of the MARS by caspase 7 [77] releases its $C$-terminal domain known as EMAP II (endothelial monocyte-activating polypeptide II) a tumor-derived cytokine that regulates angiogenesis [78,119].

Some components of the MARS are thought to regulate other cellular functions, but it is not clear whether the MARS-associated or -dissociated form of the protein is involved, or the mechanisms of 
complex-release are not determined. For instance, LeuRS has been reported to be an important regulator of one of the major pathway of cellular homeostasis through the leucine-dependent control of mTORC1 activity [120], but the relationship between MARS-LeuRS and mTORC1-related LeuRS is not known. LeuRS was described to act as a GTPase-activating protein for Rag GTPases that activate mTORC1 in response to amino acid availability [120], but these effects were not recapitulated in another study [121]. GlnRS may have an anti-apoptotic function through its glutamine-dependent interaction with apoptosis signal-regulating kinase 1 (ASK1), but the molecular species of GlnRS involved in this pathway is not known [122].

\section{Concluding Remarks}

\subsection{Origin of the MARS of the Eukaryotic-Type}

In archaea, the multi-synthetase complexes are characterized by the absence of non-synthetase scaffold proteins. The majority but not all of the components found so far associated within the complexes described in Fungi, Trypanosoma or Apicomplexa are also recovered in Eumetazoa, and contain accessory proteins with scaffolding properties. The finding that composition of the MARS recovered in different species is not the result of serial addition of components suggests that at least during the early steps of emergence, evolution of MARS followed a non-linear scheme of trials and errors before establishment of more universal rules of assembly, as observed in Bilateria. However, even in Bilateria the complex isolated in $C$. elegans casts some doubts about the universality of the MARS in higher eukaryotes. However, the finding that essentially the same MARS is present in Deuterostomia and Protostomia (Arthropoda) suggests that it pre-existed in the last common ancestor of Bilateria. The atypical MARS isolated from $C$. elegans could be the result of genome rearrangements occurring in a limited set of Nematoda belonging to the Rhabditina branch, as also proposed for evolution of GluProRS [55,57]. In Haemonchus contortus, Necator americanus or Ancylostoma ceylanicum, three nematodes from the Rhabditina branch of Nematoda, GluRS and ProRS are encoded by distinct genes, as in C. elegans. It could be the result of a fission event of the GluProRS gene appearing in a limited set of species. Indeed, in the two very closely related nematodes from the Spirurina branch of Nematoda, Brugia malayi and Loa loa, GluRS and ProRS are fused on a single gene, as also observed in Trichuris suis or Trichinella spiralis, two nematodes from the more distantly related branch of Enopla. The fission of GluProRS and MARS rearrangement could be linked. Because a bona fide GluProRS was also observed in the cnidarian sea anemone Nematostella vectensis [55], this suggests that a MARS similar to that observed in Deuterostoma could exist in Cnidaria, which would locate the origin of the eukaryotic-type MARS near the origin of Metazoan.

\subsection{Understanding the Balance between Translational and Non-Translational Functions}

The balance between their translational and noncanonical functions very often involves dissociation of components of the MARS and their association with alternative partners. The knowledge of the protein interfaces involved in the different facets of their activity is of fundamental importance as far as their connection with various physiological disorders and diseases is concerned. Indeed, it should be 
kept in mind that an intricate interaction network makes it more difficult to design molecules capable of inhibiting a single pathway.

Cellular homeostasis requires a tight control of the various functions of aminoacyl-tRNA synthetases in translational and nontranslational processes. Obviously, overexpression of a synthetase or drastic perturbation of cellular equilibrium after siRNA silencing of these essential proteins, are likely to induce responses which may not be physiologically relevant.

LysRS is the component of the MARS for which most noncanonical functions have been described in human [7]. When it accomplishes its translational function, the catalytic domain of LysRS interacts with p38 within the MARS. On the other hand, LysRS dissociates from the cytoplasmic MARS to regulate the activity of the transcription factor MITF in the nucleus [114]. LysRS has been reported to interact with several proteins: with synthenin-1 which modulates the activity of LysRS [123], with the laminin receptor which controls the stability of the receptor [118], and with the Gag polyprotein of HIV-1 for packaging tRNA ${ }_{3}{ }^{\text {Lys }}$ into the virions [124]. The mitochondrial and cytoplasmic species of LysRS are encoded by the same gene and share the same catalytic and anticodon-binding domains. The conserved region of mitochondrial LysRS interacts with mutant SOD1 in some cases of amyotrophic lateral sclerosis [125], or with the GagPol polyprotein of HIV-1 to package tRNA ${ }_{3}^{\text {Lys }}$ into viral particles [94]. It is not known whether the same surface area of LysRS is involved in the interaction with p38 and with all these secondary partners. LysRS can bind non competitively to MARS and to GagPol, suggesting that these binding sites are independent [94]. In that case, it is conceivable to isolate an inhibitor of the association between LysRS and GagPol that would not impair association of LysRS into the MARS and would not be toxic for the translation process. The LysRS:p38 interface has been described at the atomic level, from the co-crystal structure of LysRS with a non-structured peptide corresponding to the $N$-terminus of p38. It remains to be established whether association of LysRS with the native, full-length scaffold protein will reveal a similar interaction pattern.

\subsection{Perspectives}

During the past few years, understanding the function of aminoacyl-tRNA synthetases appeared to be a task much more complicated than previously anticipated due to the numerous secondary, noncanonical functions that are performed by this family of enzymes. Association and dissociation of the components of the MARS seems to be an important checkpoint for many cellular pathways. The recent finding that splice-variant synthetases may fulfill functions independently of their primary role in translation, also unexpectedly expands the sphere of influence of this family of enzymes [126].

\section{Acknowledgments}

We would like to acknowledge the contributions of the many works that could not be cited in this review due to space limitation. The research carried out in our laboratory was supported by grants from the Centre National de la Recherche Scientifique (CNRS) and the Agence nationale de recherches sur le sida et les hépatites virales (ANRS). 


\section{Conflicts of Interest}

The authors declare no conflict of interest.

\section{References}

1. Ibba, M.; Söll, D. Aminoacyl-tRNA synthesis. Annu. Rev. Biochem. 2000, 69, 617-650.

2. Schimmel, P.; Söll, D. Aminoacyl-tRNA synthetases: General features and recognition of transfer RNAs. Annu. Rev. Biochem. 1979, 48, 601-648.

3. Guo, M.; Schimmel, P.; Yang, X.L. Functional expansion of human tRNA synthetases achieved by structural inventions. FEBS Lett. 2010, 584, 434-442.

4. Guo, M.; Yang X.L.; Schimmel, P. New functions of aminoacyl-tRNA synthetases beyond translation. Nat. Rev. Mol. Cell Biol. 2011, 11, 668-674.

5. Hausmann, C.D.; Ibba, M. Aminoacyl-tRNA synthetase complexes: Molecular multitasking revealed. FEMS Microbiol. Rev. 2008, 32, 705-721.

6. Guo, M.; Schimmel, P. Essential nontranslational functions of tRNA synthetases. Nat. Chem. Biol. 2013, 9, 145-153.

7. Motzik, A.; Nechushtan, H.; Foo, S.Y.; Razin, E. Non-canonical roles of lysyl-tRNA synthetase in health and disease. Trends Mol. Med. 2013, 19, 726-731.

8. Yao, P.; Fox, P.L. Aminoacyl-tRNA synthetases in medicine and disease. EMBO Mol. Med. 2013, 5, 332-343.

9. An, S.; Musier Forsyth, K. Cys-tRNA ${ }^{\text {Pro }}$ editing by Haemophilus influenzae YbaK via a novel synthetase-YbaK-tRNA ternary complex. J. Biol. Chem. 2005, 280, 34465-34472.

10. Lipman, R.S.A.; Chen, J.; Evilia, C.; Vitseva, O.; Hou, Y.M. Association of an aminoacyl-tRNA synthetase with a putative metabolic protein in archaea. Biochemistry 2003, 42, 7487-7496.

11. Oza, J.P.; Sowers, K.R.; Perona, J.J. Linking energy production and protein synthesis in hydrogenotrophic methanogens. Biochemistry 2012, 51, 2378-2389.

12. Godinic, V.; Mocibob, M.; Rocak, S.; Ibba, M.; Weygand-Durasevic, I. Peroxin Pex21p interacts with the $C$-terminal noncatalytic domain of yeast seryl-tRNA synthetase and forms a specific ternary complex with tRNA ${ }^{\text {Ser }}$. FEBS J. 2007, 274, 2788-2799.

13. Laporte, D.; Huot, J.L.; Bader, G.; Enkler, L.; Senger, B.; Becker, H.D. Exploring the evolutionary diversity and assembly modes of multi-aminoacyl-tRNA synthetase complexes: Lessons from unicellular organisms. FEBS Lett. 2014, 588, 4268-4278.

14. Harris, C.L. An aminoacyl-tRNA synthetase complex in Escherichia coli. J. Bacteriol. 1987, 169, 2718-2723.

15. Harris, C.L.; Kolanko, C.J. Aminoacyl-tRNA synthetase complex in Saccharomyces cerevisiae. Biochem. J. 1995, 309, 321-324.

16. Bandyopadhyay, A.K.; Deutscher, M.P. Complex of aminoacyl-tRNA synthetases. J. Mol. Biol. 1971, 60, 113-122.

17. Kaminska, M.; Havrylenko, S.; Decottignies, P.; Le Marechal, P.; Negrutskii, B.; Mirande, M. Dynamic organization of aminoacyl-tRNA synthetase complexes in the cytoplasm of human cells. J. Biol. Chem. 2009, 284, 13746-13754. 
18. Barbarese, E.; Koppel, D.E.; Deutscher, M.P.; Smith, C.L.; Ainger, K.; Morgan, F.; Carson, J.H. Protein translation components are colocalized in granules in oligodendrocytes. J. Cell Sci. 1995, 108, 2781-2790.

19. Negrutskii, B.S.; Stapulionis, R.; Deutscher, M.P. Supramolecular organization of the mammalian translation system. Proc. Natl. Acad. Sci. USA 1994, 91, 964-968.

20. Praetorius-Ibba, M.; Hausmann, C.D.; Paras, M.; Rogers, T.E.; Ibba, M. Functional association between three archaeal aminoacyl-tRNA synthetases. J. Biol. Chem. 2007, 282, 3680-3687.

21. Hausmann, C.D.; Ibba, M. Structural and functional mapping of the archaeal multi-aminoacyl-tRNA synthetase complex. FEBS Lett. 2008, 582, 2178-2182.

22. Hausmann, C.D.; Praetorius-Ibba, M.; Ibba, M. An aminoacyl-tRNA synthetase:Elongation factor complex for substrate channeling in archaeal translation. Nucleic Acids Res. 2007, 35, 6094-6102.

23. Godinic-Mikulcic, V.; Jaric, J.; Hausmann, C.D.; Ibba, M.; Weygand-Durasevic, I. An archaeal tRNA-synthetase complex that enhances aminoacylation under extreme conditions. J. Biol. Chem. 2011, 286, 3396-3404.

24. Godinic-Mikulcic, V.; Jaric, J.; Greber, B.J.; Franke, V.; Hodnik, V.; Anderluh, G.; Ban, N.; Weygand-Durasevic, I. Archaeal aminoacyl-tRNA synthetases interact with the ribosome to recycle tRNAs. Nucleic Acids Res. 2014, 42, 5191-5201.

25. Raina, M.; Elgamal, S.; Santangelo, T.J.; Ibba, M. Association of a multi-synthetase complex with translating ribosomes in the archaeon Thermococcus kodakarensis. FEBS Lett. 2012, 586, 2232-2238.

26. Simos, G.; Segref, A.; Fasiolo, F.; Hellmuth, K.; Shevchenko, A.; Mann, M.; Hurt, E.C. The yeast protein Arc1p binds to tRNA and functions as a cofactor for the methionyl- and glutamyl-tRNA synthetases. EMBO J. 1996, 15, 5437-5448.

27. Simos, G.; Sauer, A.; Fasiolo, F.; Hurt, E.C. A conserved domain within Arc1p delivers tRNA to aminoacyl-tRNA synthetases. Mol. Cell 1998, 1, 235-242.

28. Golinelli-Cohen, M.P.; Mirande, M. Arc1p is required for cytoplasmic confinement of synthetases and tRNA. Mol. Cell. Biochem. 2007, 300, 47-59.

29. Graindorge, J.S.; Senger, B.; Tritch, D.; Simos, G.; Fasiolo, F. Role of Arc1p in the modulation of yeast glutamyl-tRNA synthetase activity. Biochemistry 2005, 44, 1344-1352.

30. Golinelli-Cohen, M.P.; Zakrzewska, A.; Mirande, M. Complementation of yeast Arc1p by the p43 component of the human multisynthetase complex does not require its association with yeast MetRS and GluRS. J. Mol. Biol. 2004, 340, 15-27.

31. Galani, K.; Grosshans, H.; Deinert, K.; Hurt, E.C.; Simos, G. The intracellular location of two aminoacyl-tRNA synthetases depends on complex formation with Arclp. EMBO J. 2001, 20, 6889-6898.

32. Frechin, M.; Enkler, L.; Tetaud, E.; Laporte, D.; Senger, B.; Blancard, C.; Hammann, P.; Bader, G.; Clauder-Münster, S.; Steinmetz, L.M.; et al. Expression of nuclear and mitochondrial genes encoding ATP synthase is synchronized by disassembly of a multisynthetase complex. Mol. Cell 2014, 56, 763-776. 
33. Frechin, M.; Senger, B.; Braye, M.; Kern, D.; Martin, R.P.; Becker, H.D. Yeast mitochondrial Gln-tRNA $^{\text {Gln }}$ is generated by a GatFAB-mediated transamidation pathway involving Arc1p-controlled subcellular sorting of cytosolic GluRS. Genes Dev. 2009, 23, 1119-1130.

34. Simader, H.; Hothorn, M.; Kohler, C.; Basquin, J.; Simos, G.; Suck, D. Structural basis of yeast aminoacyl-tRNA synthetase complex formation revealed by crystal structures of two binary sub-complexes. Nucleic Acids Res. 2006, 34, 3968-3979.

35. Karanasios, E.; Simader, H.; Panayotou, G.; Suck, D.; Simos, G. Molecular determinants of the yeast Arc1p-aminoacyl-tRNA synthetase complex assembly. J. Mol. Biol. 2007, 374, 1077-1090.

36. Kim, H.S.; Hoja, U.; Stolz, J.; Sauer, G.; Schweizer, E. Identification of the tRNA-binding protein Arc1p as a novel target of in vivo biotinylation in Saccharomyces cerevisiae. J. Biol. Chem. 2004, 279, 42445-42452.

37. Koehler, C.; Round, A.; Simader, H.; Suck, D.; Svergun, D. Quaternary structure of the yeast Arc1p-aminoacyl-tRNA synthetase complex in solution and its compaction upon binding of tRNAs. Nucleic Acids Res. 2013, 41, 667-676.

38. Van Rooyen, J.M.; Murat, J.B.; Hammoudi, P.M.; Kieffer-Jaquinod, S.; Coute, Y.; Sharma, A.; Pelloux, H.; Belrhali, H.; Hakimi, M.A. Assembly of the novel five-component apicomplexan multi-aminoacyl-tRNA synthetase complex is driven by the hybrid scaffold protein Tg-p43. PLoS One 2014, 9, e89487.

39. Cestari, I.; Kalidas, S.; Monnerat, S.; Anupama, A.; Phillips, M.A.; Stuart, K. A multiple aminoacyl-tRNA synthetase complex that enhances tRNA-aminoacylation in African trypanosomes. Mol. Cell. Biol. 2013, 33, 4872-4888.

40. Vennegoor, C.; Bloemendal, H. Occurrence and particle character of aminoacyl-tRNA synthetases in the post-microsomal fraction from rat liver. Eur. J. Biochem. 1972, 26, 462-473.

41. Som, K.; Hardesty, B. Isolation and partial characterization of an aminoacyl-tRNA synthetase complex from rabbit reticulocytes. Arch. Biochem. Biophys. 1975, 166, 507-517.

42. Denney, R.M. Detection and partial purification of rapidly sedimenting forms of aminoacyl-transfer ribonucleic acid synthetases from human placenta. Arch. Biochem. Biophys. 1977, 183, 156-167.

43. Ussery, M.A.; Tanaka, W.K.; Hardesty, B. Subcellular distribution of aminoacyl-tRNA synthetases in various eukaryotic cells. Eur. J. Biochem. 1977, 72, 491-500.

44. Kellermann, O.; Brevet, A.; Tonetti, H.; Waller, J.P. Macromolecular complexes of aminoacyl-tRNA synthetases from eukaryotes. Extensive purification and characterization of the high-molecular-weight complex(es) of seven aminoacyl-tRNA synthetases from sheep liver. Eur. J. Biochem. 1979, 99, 541-550.

45. Johnson, D.L.; Yang, D.C.H. Stoichiometry and composition of an aminoacyl-tRNA synthetase complex from rat liver. Proc. Natl. Acad. Sci. USA 1981, 78, 4059-4062.

46. Kellermann, O.; Tonetti, H.; Brevet, A.; Mirande, M.; Pailliez, J.P.; Waller, J.P. Macromolecular complexes from sheep and rabbit containing seven aminoacyl-tRNA synthetases I. Species specificity of the polypeptide composition. J. Biol. Chem. 1982, 257, 11041-11048.

47. Godar, D.E.; Godar, D.E.; Garcia, V.; Jacobo, A.; Aebi, U.; Yang, D.C.H. Structural organization of the multienzyme complex of mammalian aminoacyl-tRNA synthetases. Biochemistry 1988, $27,6921-6928$. 
48. Mirande, M.; Le Corre, D.; Waller, J.P. A complex from cultured Chinese hamster ovary cells containing nine aminoacyl-tRNA synthetases. Thermolabile leucyl-tRNA synthetase from the tsH1 mutant cell line is an integral component of this complex. Eur. J. Biochem. 1985, 147, 281-289.

49. Norcum, M.T. Isolation and electron microscopic characterization of the high molecular mass aminoacyl-tRNA synthetase complex from murine erythroleukemia cells. J. Biol. Chem. 1989, 264, 15043-15051.

50. Quevillon, S.; Mirande, M. The p18 component of the multisynthetase complex shares a protein motif with the $b$ and $g$ subunits of eukaryotic elongation factor 1. FEBS Lett. 1996, 395, 63-67.

51. Quevillon, S.; Robinson, J.C.; Berthonneau, E.; Siatecka, M.; Mirande, M. Macromolecular assemblage of aminoacyl-tRNA synthetases: Identification of protein-protein interactions and characterization of a core protein. J. Mol. Biol. 1999, 285, 183-195.

52. Quevillon, S.; Agou, F.; Robinson, J.C.; Mirande, M. The p43 component of the mammalian multi-synthetase complex is likely to be the precursor of the endothelial monocyte-activating polypeptide II cytokine. J. Biol. Chem. 1997, 272, 32573-32579.

53. Cérini, C.; Kerjan, P.; Astier, M.; Gratecos, D.; Mirande, M.; Semeriva, M. A component of the multisynthetase complex is a multifunctional aminoacyl-tRNA synthetase. EMBO J. 1991, 10, 4267-4277.

54. Kerjan, P.; Triconnet, M.; Waller, J.P. Mammalian prolyl-tRNA synthetase corresponds to the $150 \mathrm{kDa}$ subunit of the high-M(r) aminoacyl-tRNA synthetase complex. Biochimie 1992, 74, 195-205.

55. Ray, P.S.; Sullivan, J.C.; Jia, J.; Francis, J.; Finnerty, J.R.; Fox, P.L. Evolution of function of a fused metazoan tRNA synthetase. Mol. Biol. Evol. 2011, 28, 437-447.

56. Berthonneau, E.; Mirande, M. A gene fusion event in the evolution of aminoacyl-tRNA synthetases. FEBS Lett. 2000, 470, 300-304.

57. Ray, P.S.; Fox, P.L. Origin and evolution of glutamyl-prolyl tRNA synthetase WHEP domains reveal evolutionary relationships within Holozoa. PLoS One 2014, 9, e98493.

58. Dias, J.; Renault, L.; Perez, J.; Mirande, M. Small-angle X-ray solution scattering study of the multi-aminoacyl-tRNA synthetase complex reveals an elongated and multi-armed particle. J. Biol. Chem. 2013, 288, 23979-23989.

59. Zheng, Y.G.; Wei, H.; Ling, C.; Xu, M.G.; Wang, E.D. Two forms of human cytoplasmic arginyl-tRNA synthetase produced from two translation initiations by a single mRNA. Biochemistry 2006, 45, 1338-1344.

60. Lazard, M.; Mirande, M. Cloning and analysis of a cDNA encoding mammalian arginyl-tRNA synthetase, a component of the multisynthetase complex with a hydrophobic $N$-terminal extension. Gene 1993, 132, 237-245.

61. Kyriacou, S.V.; Deutscher, M.P. An important role for the multienzyme aminoacyl-tRNA synthetase complex in mammalian translation and cell growth. Mol. Cell 2008, 29, 419-427.

62. Sivaram, P.; Deutscher, M.P. Existence of two forms of rat liver arginyl-tRNA synthetase suggests channeling of aminoacyl-tRNA for protein synthesis. Proc. Natl. Acad. Sci. USA 1990, $87,3665-3669$. 
63. Yao, P.; Potdar, A.A.; Arif, A.; Ray, P.S.; Mukhopadhyay, R.; Willard, B.; Xu, Y.; Yan, J.; Saidel, G.M.; Fox, P.L. Coding region polyadenylation generates a truncated tRNA synthetase that counters translation repression. Cell 2012, 149, 88-100.

64. Tolkunova, E.; Park, H.; Xia, J.; King, M.P.; Davidson, E. The human lysyl-tRNA synthetase gene encodes both the cytoplasmic and mitochondrial enzymes by means of an unusual alternative splicing of the primary transcript. J. Biol. Chem. 2000, 275, 35063-35069.

65. Kaminska, M.; Shalak, V.; Francin, M.; Mirande, M. Viral hijacking of mitochondrial lysyl-tRNA synthetase. J. Virol. 2007, 81, 68-73.

66. Wolfson, A.; Knight, R. Occurrence of the aminoacyl-tRNA synthetases in high-molecular weight complexes correlates with the size of substrate amino acids. FEBS Lett. 2005, 579, 3467-3472.

67. Eswarappa, S.M.; Fox, P.L. Citric acid cycle and the origin of MARS. Trends Biochem. Sci. 2013, 38, 222-228.

68. Ahn, H.C.; Kim, S.; Lee, B.J. Solution structure and p43 binding of the p38 leucine zipper motif: Coiled-coil interactions mediate the association between $\mathrm{p} 38$ and p43. FEBS Lett. 2003, 542, 119-124.

69. Choi, J.W.; Kim, D.G.; Lee, A.E.; Kim, H.R.; Lee, J.Y.; Kwon, N.H.; Shin, Y.K.; Hwang, S.K.; Chang, S.H.; Cho, M.H.; et al. Cancer-associated splicing variant of tumor suppressor AIMP2/p38: Pathological implication in tumorigenesis. PLoS Genet. 2011, 7, e1001351.

70. Kaminska, M.; Havrylenko, S.; Decottignies, P.; Gillet, S.; Marechal, P.L.; Negrutskii, B.; Mirande, M. Dissection of the structural organization of the aminoacyl-tRNA synthetase complex. J. Biol. Chem. 2009, 284, 6053-6060.

71. Kim, J.Y.; Kang, Y.S.; Lee, J.W.; Kim, H.J.; Ahn, Y.H.; Park, H.; Ko, Y.G.; Kim, S. p38 is essential for the assembly and stability of macromolecular tRNA synthetase complex: Implications for its physiological significance. Proc. Natl. Acad. Sci. USA 2002, 99, 7912-7916.

72. Corti, O.; Hampe, C.; Koutnikova, H.; Darios, F.; Jacquier, S.; Prigent, A.; Robinson, J.C.; Pradier, L.; Ruberg, M.; Mirande, M.; et al. The p38 subunit of the aminoacyl-tRNA synthetase complex is a Parkin substrate: Linking protein biosynthesis and neurodegeneration. Hum. Mol. Genet. 2003, 12, 1427-1437.

73. Ko, H.S.; von Coelln, R.; Sriram, S.R.; Kim, S.W.; Chung, K.K.K.; Pletnikova, O.; Troncoso, J.; Johnson, B.; Saffary, R.; Goh, E.L.; et al. Accumulation of the authentic parkin substrate aminoacyl-tRNA synthetase cofactor, p38/JTV-1, leads to catecholaminergic cell death. J. Neurosci. 2005, 25, 7968-7978.

74. Sriram, S.R.; Li, X.J.; Ko, H.S.; Chung, K.K.K.; Wong, E.; Lim, K.L.; Dawson, V.L.; Dawson, T.M. Familial-associated mutations differentially disrupt the solubility, localization, binding and ubiquitination properties of parkin. Hum. Mol. Genet. 2005, 14, 2571-2586.

75. Kim, K.J.; Park, M.C.; Choi, S.J.; Oh, Y.S.; Choi, E.C.; Cho, H.J.; Kim, M.H.; Kim, S.H.; Kim, D.W.; Kim, S.; et al. Determination of three-dimensional structure and residues of the novel tumor suppressor AIMP3/p18 required for the interaction with ATM. J. Biol. Chem. 2008, 283, 14032-14040. 
76. Kang, T.; Kwon, N.H.; Lee, J.Y.; Park, M.C.; Kang, E.; Kim, H.H.; Kang, T.J.; Kim, S. AIMP3/p18 controls translational initiation by mediating the delivery of charged initiator tRNA to initiation complex. J. Mol. Biol. 2012, 423, 475-481.

77. Shalak V.; Kaminska M.; Mitnacht-Kraus R.; Vandenabeele P.; Clauss M.; Mirande M. The EMAPII cytokine is released from the mammalian multisynthetase complex after cleavage of its p43/proEMAPII component. J. Biol. Chem. 2001, 276, 23769-23776.

78. Kao, J.; Ryan, J.; Brett, G.; Chen, J.; Shen, H.; Fan, Y.G.; Godman, G.; Familletti, P.C.; Wang, F.; Pan, Y.C.; et al. Endothelial monocyte-activating polypeptide II. A novel tumor-derived polypeptide that activates host-response mechanisms. J. Biol. Chem. 1992, 267, 20239-20247.

79. Swairjo, M.A.; Morales, A.J.; Wang, C.C.; Ortiz, A.R.; Schimmel, P. Crystal structure of Trbp111: A structure-specific tRNA-binding protein. EMBO J. 2000, 19, 6287-6298.

80. Renault, L.; Kerjan, P.; Pasqualato, S.; Menetrey, J.; Robinson, J.C.; Kawaguchi, S.; Vassylyev, D.G.; Yokoyama, S.; Mirande, M.; Cherfils, J. Structure of the EMAPII domain of human aminoacyl-tRNA synthetase complex reveals evolutionary dimer mimicry. EMBO J. 2001, 20, 570-578.

81. Kim, Y.; Shin, J.; Li, R.B.; Cheong, C.; Kim, K.; Kim, S. A novel anti-tumor cytokine contains an RNA binding motif present in aminoacyl-tRNA synthetases. J. Biol. Chem. 2000, 275, 27062-27068.

82. Park, S.G.; Jung, K.H.; Lee, J.S.; Jo, Y.J.; Motegi, H.; Kim, S.; Shiba, K. Precursor of pro-apoptotic cytokine modulates aminoacylation activity of tRNA synthetase. J. Biol. Chem. 1999, 274, 16673-16676.

83. Guigou, L.; Shalak, V.; Mirande, M. The tRNA-interacting factor p43 associates with mammalian arginyl-tRNA synthetase but does not modify its tRNA aminoacylation properties. Biochemistry 2004, 43, 4592-4600.

84. Shalak, V.; Kaminska, M.; Mirande, M. Translation initiation from two in-frame AUGs generates mitochondrial and cytoplasmic forms of the p43 component of the multisynthetase complex. Biochemistry 2009, 48, 9959-9968.

85. Norcum, M.T.; Warrington, J.A. Structural analysis of the multienzyme aminoacyl-tRNA synthetase complex: A three-domain model based on reversible chemical crosslinking. Protein Sci. 1998, 7, 79-87.

86. Rho, S.B.; Kim, M.J.; Lee, J.S.; Seol, W.G.; Motegi, H.; Kim, S.; Shiba, K. Genetic dissection of protein-protein interactions in multi-tRNA synthetase complex. Proc. Natl. Acad. Sci. USA 1999, 96, 4488-4493.

87. Ling, C.; Yao, Y.N.; Zheng, Y.G.; Wei, H.; Wang, L.; Wu, X.F.; Wang, E.D. The $C$-terminal appended domain of human cytosolic leucyl-tRNA synthetase is indispensable in its interaction with arginyl-tRNA synthetase in the multi-tRNA synthetase complex. J. Biol. Chem. 2005, 280, 34755-34763.

88. Rho, S.B.; Lee, K.H.; Kim, J.W.; Shiba, K.; Jo, Y.J.; Kim, S. Interaction between human tRNA synthetases involves repeated sequence elements. Proc. Natl. Acad. Sci. USA 1996, 93, 10128-10133. 
89. Robinson, J.C.; Kerjan, P.; Mirande, M. Macromolecular assemblage of aminoacyl-tRNA synthetases: Quantitative analysis of protein-protein interactions and mechanism of complex assembly. J. Mol. Biol. 2000, 304, 983-994.

90. He, R.; Zu, L.D.; Yao, P.; Chen, X.; Wang E.D. Two non-redundant fragments in the $N$-terminal peptide of human cytosolic methionyl-tRNA synthetase were indispensable for the multi-synthetase complex incorporation and enzyme activity. Biochim. Biophys. Acta 2009, 1794, 347-354.

91. Mirande, M.; Kellermann, O.; Waller, J.P. Macromolecular complexes from sheep and rabbit containing seven aminoacyl-tRNA synthetases. II. Structural characterization of the polypeptide components and immunological identification of the methionyl-tRNA synthetase subunit. J. Biol. Chem. 1982, 257, 11049-11055.

92. Kaminska, M.; Shalak, V.; Mirande, M. The appended C-domain of human methionyl-tRNA synthetase has a tRNA-sequestering function. Biochemistry 2001, 40, 14309-14316.

93. Kim, T.; Park, S.G.; Kim, J.E.; Seol, W.; Ko, Y.G.; Kim, S. Catalytic peptide of human glutaminyl-tRNA synthetase is essential for its assembly to the aminoacyl-tRNA synthetase complex. J. Biol. Chem. 2000, 275, 21768-21772.

94. Kobbi, L.; Octobre, G.; Dias, J.; Comisso, M.; Mirande, M. Association of mitochondrial lysyl-tRNA synthetase with HIV-1 GagPol involves catalytic domain of the synthetase and transframe and integrase domains of Pol. J. Mol. Biol. 2011, 410, 875-886.

95. Ofir-Birin, Y.; Fang, P.; Bennett, S.P.; Zhang, H.M.; Wang, J.; Rachmin, I.; Shapiro, R.; Song, J.; Dagan, A.; Pozo, J.; et al. Structural switch of lysyl-tRNA synthetase between translation and transcription. Mol. Cell 2013, 49, 30-42.

96. Fu, Y.; Kim, Y.; Jin, K.S.; Kim, H.S.; Kim, J.H.; Wang, D.; Park, M.; Jo, C.H.; Kwon, N.H.; Kim, D.; et al. Structure of the ArgRS-GlnRS-AIMP1 complex and its implications for mammalian translation. Proc. Natl. Acad. Sci. USA 2014, 111, 15084-15089.

97. Lazard, M.; Kerjan, P.; Agou, F.; Mirande, M. The tRNA-dependent activation of arginine by arginyl-tRNA synthetase requires inter-domain communication. J. Mol. Biol. 2000, 302, 991-1004.

98. Norcum, M.T.; Boisset, N. Three-dimensional architecture of the eukaryotic multisynthetase complex determined from negatively stained and cryoelectron micrographs. FEBS Lett. 2002, 512, 298-302.

99. Kerjan, P.; Cérini, C.; Semeriva, M.; Mirande, M. The multienzyme complex containing nine aminoacyl-tRNA synthetases is ubiquitous from Drosophila to mammals. Biochim. Biophys. Acta 1994, 1199, 293-297.

100. Brandsma, M.; Kerjan, P.; Dijk, J.; Janssen, G.M.; Moller, W. Valyl-tRNA synthetase from Artemia. Purification and association with elongation factor 1. Eur. J. Biochem. 1995, 233, 277-282.

101. Havrylenko, S.; Legouis, R.; Negrutskii, B.; Mirande, M. Caenorhabditis elegans evolves a new architecture for the multi-aminoacyl-tRNA synthetase complex. J. Biol. Chem. 2011, 286, 28476-28487.

102. Havrylenko, S.; Legouis, R.; Negrutskii, B.; Mirande, M. Methionyl-tRNA synthetase from Caenorhabditis elegans. A specific multi-domain organization for convergent functional evolution. Protein Sci. 2010, 19, 2475-2484. 
103. Motorin, Y.A.; Wolfson, A.D.; Orlovsky, A.F.; Gladilin, K.L. Mammalian valyl-tRNA synthetase forms a complex with the first elongation factor. FEBS Lett. 1988, 238, 262-264.

104. Bec, G.; Kerjan, P.; Zha, X.D.; Waller, J.P. Valyl-tRNA synthetase from rabbit liver. I. Purification as a heterotypic complex in association with elongation factor 1. J. Biol. Chem. 1989, 264, 21131-21137.

105. Mansilla, F.; Friis, I.; Jadidi, M.; Nielsen, K.M.; Clark, B.F.C.; Knudsen, C.R. Mapping the human translation elongation factor eEF1H complex using the yeast two-hybrid system. Biochem. J. 2002, 365, 669-676.

106. Bec, G.; Kerjan, P.; Waller, J.P. Reconstitution in vitro of the valyl-tRNA synthetase-elongation factor (EF) $1 \beta \gamma \delta$ complex: Essential roles of the $\mathrm{NH}_{2}$-terminal extension of valyl-tRNA synthetase and of the EF-1 $\delta$ subunit in complex formation. J. Biol. Chem. 1994, 269, 2086-2092.

107. Reed, V.S.; Wastney, M.E.; Yang, D.C.H. Mechanisms of the transfer of aminoacyl-tRNA from aminoacyl-tRNA synthetase to the elongation factor 1 $\alpha$. J. Biol. Chem. 1994, 269, 32932-32936.

108. David, A.; Netzer, N.; Strader, M.B.; Das, S.R.; Chen, C.Y.; Gibbs, J.; Pierre, P.; Bennink, J.R.; Yewdell, J.W. RNA binding targets aminoacyl-tRNA synthetases to translating ribosomes. J. Biol. Chem. 2011, 286, 20688-20700.

109. Stapulionis, R.; Kolli, S.; Deutscher, M.P. Efficient mammalian protein synthesis requires an intact F-Actin system. J. Biol. Chem. 1997, 272, 24980-24986.

110. Stapulionis, R.; Deutscher, M.P. A channeled tRNA cycle during mammalian protein synthesis. Proc. Natl. Acad. Sci. USA 1995, 92, 7158-7161.

111. Mirande, M. Processivity of translation in the eukaryote cell: Role of aminoacyl-tRNA synthetases. FEBS Lett. 2010, 584, 443-447.

112. Ray, P.S.; Arif, A.; Fox, P.L. Macromolecular complexes as depots for releasable regulatory proteins. Trends Biochem. Sci. 2007, 32, 158-164.

113. Lee, Y.N.; Nechushtan, H.; Figov, N.; Razin, E. The function of lysyl-tRNA synthetase and Ap4A as signaling regulators of MITF activity in FceRI-activated mast cells. Immunity 2004, 20, 145-151.

114. Yannay-Cohen, N.; Carmi-Levy, I.; Kay, G.; Yang, C.M.; Han, J.M.; Kemeny, D.M.; Kim, S.; Nechushtan, H.; Razin, E. LysRS serves as a key signaling molecule in the immune response by regulating gene expression. Mol. Cell 2009, 34, 603-611.

115. Kisselev, L.L.; Justesen, J.; Wolfson, A.D.; Frolova, L.Y. Diadenosine oligophosphates (Ap $\mathrm{A}_{n}$ ), a novel class of signalling molecules? FEBS Lett. 1998, 427, 157-163.

116. Sampath, P.; Mazumder, B.; Seshadri, V.; Gerber, C.A.; Chavatte, L.; Kinter, M.; Ting, S.M.; Dignam, J.D.; Kim, S.; Driscoll, D.M.; et al. Noncanonical function of glutamyl-prolyl-tRNA synthetase: Gene-specific silencing of translation. Cell 2004, 119, 195-208.

117. Arif, A.; Jia, J.; Mukhopadhyay, R.; Willard, B.; Kinter, M.; Fox, P.L. Two-site phosphorylation of EPRS coordinates multimodal regulation of noncanonical translational control activity. Mol. Cell 2009, 35, 164-180.

118. Kim, D.G.; Lee, J.Y.; Kwon, N.H.; Fang, P.; Zhang, Q.; Wang, J.; Young, N.L.; Guo, M.; Cho, H.Y.; Mushtaq, A.U.; et al. Chemical inhibition of prometastatic lysyl-tRNA synthetase-laminin receptor interaction. Nat. Chem. Biol. 2014, 10, 29-34. 
119. Park, S.G.; Kang, Y.S.; Ahn, Y.H.; Lee, S.H.; Kim, K.R.; Kim, K.W.; Koh, G.Y.; Ko, Y.G.; Kim, S. Dose-dependent biphasic activity of tRNA synthetase-associating factor, p43, in angiogenesis. J. Biol. Chem. 2002, 277, 45243-45248.

120. Han, J.M.; Jeong, S.J.; Park, M.C.; Kim, G.; Kwon, N.H.; Kim, H.K.; Ha, S.H.; Ryu, S.H.; Kim, S. Leucyl-tRNA synthetase is an intracellular leucine sensor for the mTORC1-signaling pathway. Cell 2012, 149, 410-424.

121. Tsun, Z.Y.; Bar-Peled, L.; Chantranupong, L.; Zoncu, R.; Wang, T.; Kim, C.; Spooner, E.; Sabatini, D.M. The folliculin tumor suppressor is a GAP for the RagC/D GTPases that signal amino acid levels to mTORC1. Mol. Cell 2013, 52, 495-505.

122. Ko, Y.G.; Kim, E.K.; Kim, T.; Park, H.; Park, H.S.; Choi, E.J.; Kim, S. Glutamine-dependent antiapoptotic interaction of human glutaminyl-tRNA synthetase with apoptosis signal-regulating kinase 1. J. Biol. Chem. 2001, 276, 6030-6036.

123. Meerschaert, K.; Remue, E.; de Ganck, A.; Staes, A.; Boucherie, C.; Gevaert, K.; Vandekerckhove, J.; Kleiman, L.; Gettemans, J. The tandem PDZ protein Syntenin interacts with the aminoacyl tRNA synthetase complex in a lysyl-tRNA synthetase-dependent manner. J. Proteome Res. 2008, 7, 4962-4973.

124. Javanbakht, H.; Halwani, R.; Cen, S.; Saadatmand, J.; Musier-Forsyth, K.; Gottlinger, H.; Kleiman, L. The interaction between HIV-1 Gag and human lysyl-tRNA synthetase during viral assembly. J. Biol. Chem. 2003, 278, 27644-27651.

125. Kunst, C.B.; Mezey, E.; Brownstein, M.J.; Patterson, D. Mutations in SOD1 associated with amyotrophic lateral sclerosis cause novel protein interactions. Nat. Genet. 1997, 15, 91-94.

126. Lo, W.S.; Gardiner, E.; Xu, Z.; Lau, C.F.; Wang, F.; Zhou, J.J.; Mendlein, J.D.; Nangle, L.A.; Chiang, K.P.; Yang, X.L.; et al. Human tRNA synthetase catalytic nulls with diverse functions. Science 2014, 345, 328-332.

(C) 2015 by the authors; licensee MDPI, Basel, Switzerland. This article is an open access article distributed under the terms and conditions of the Creative Commons Attribution license (http://creativecommons.org/licenses/by/4.0/). 